\title{
Travelers' ability to observe changes in traffic intensities and traffic light settings
}

\author{
Jaap Vreeswijk, Michael Do, Wilco Middag, Marieke Martens, Eric van Berkum and Bart van Arem
}

\begin{abstract}
Travel choice behavior is an important determinant in traffic and subject to human imperfection and bounded rationality. In decision-making processes travelers seldom act perfectly rational. Traffic models and traffic network management measure could become more realistic and effective, if travelers' (dis)abilities, reasoning and perception are better understood and taken into consideration. The aims of this study were: (1) to determine travelers' ability to detect changing traffic intensities and traffic light setting on their trip, and (2) to determine how travelers estimate the value of such changes. The survey results show that travelers are more sensitive to changes in traffic light settings than to changes in traffic intensities, but in general unable to observe all changes in traffic conditions. Besides, a relative large group of travelers perceive the opposite of what they actually experience.
\end{abstract}

\section{INTRODUCTION}

L imited cognitive abilities cause imperfections in traveler's choice behavior which, to a certain extent, are regarded as systematic and predictable $[1,2]$. As a result, travelers do not always behave in a rational way which leads to suboptimal decisions. However, many models of travel choice behavior used in traffic modeling are based on standard economics and assume that people are rational decision makers and above all perfectly informed about the available choice alternatives. In other words, they can calculate the value of the different options available, they are able to derive the optimal choice, and they are cognitively unhindered in weighting the implications of each potential choice [3-5]. In reality, people have limited knowledge and constrained cognitive abilities, leading to prejudiced reasoning and certain randomness in behavior and choice outcomes [1]. In contrast with standard economics,

Manuscript received August 3, 2011.

Jaap Vreeswijk is with Peek Traffic BV, Basicweg 16, 3821 BR Amersfoort, The Netherlands (corresponding author; phone: +31-33-4541724, fax: +31-33-454-1740, e-mail: jaap.vreeswijk@peektraffic.nl).

Michael Do is a graduate student in Faculty of Engineering Technology, University of Twente, Drienerlolaan 5, 7522 NB Enschede, The Netherlands (e-mail: m.do@student.utwente.nl).

Wilco Middag is a graduate student in Faculty of Engineering Technology, University of Twente, Drienerlolaan 5, 7522 NB Enschede, The Netherlands (e-mail: w.j.middag@student.utwente.nl).

Mariek Martens is with Centre for Transport Studies, Faculty of Engineering Technology, University of Twente, Drienerlolaan 5, 7522 NB Enschede, The Netherlands (e-mail: m.h.martens@ctw.utwente.nl).

Eric van Berkum is with Centre for Transport Studies, Faculty of Engineering Technology, University of Twente, Drienerlolaan 5, $7522 \mathrm{NB}$ Enschede, The Netherlands (e-mail: e.c.vanberkum@ctw.utwente.nl).

Bart van Arem is with Department of Transport and Planning, Delft University of Technology, Stevinweg 1, 2628 CN Delft, The Netherlands (e-mail: b.vanarem@tudelft.nl). behavioral economics draw on the aspects of both (cognitive) psychology and economics, and study the motives and behaviors that explain deviations from rational behavior [8-9]. It is not just the behavior (i.e. choice outcome) that is of interest, but also the decision-making process behind such behavior. Irrational behavior is about human imperfection and the gap between human and rational behavior. Recent studies provide evidence that such behaviors are systematic, consistent, repetitive, and therefore predictable $[1,2]$.

Using this knowledge on human imperfection and bounded rationality provides opportunities for the effective operation of traffic network management as stated by the concept of 'regulation flexibility' [6]. This concept tries to take advantage of traveler's (in)ability to respond to some change in the attributes of their trip or the traffic system, in contrast to the many research that aims to compensate for people's failure to detect changes as a result of change blindness $[7,8]$. Regulation flexibility assumes that changes resulting from traffic management measures and which remain below a certain threshold do not affect the acceptance of travelers nor lead to significant behavioral response. The resulting margin allows road operators to adjust the settings of traffic control systems, without travelers noticing them and causing undesirable side effects of the control measure. If possible, such uncontrolled side effects should be prevented at all times as day-to-day traffic equilibriums not necessarily return to the same state when a change is first introduced and later removed [9]. A concept like regulation flexibility relies heavily on the predictability of travel choice behavior based on empirical data. However, in order to develop better descriptive models of travel choice behavior and validate theories derived from behavioral economics; more empirical research is needed $[1,2,5,10]$.

In this paper, the decision-making process is regarded as a process consisting of three successive stages: 'observation', 'evaluation' and 'choice'. A conceptual framework is discussed in [6], which illustrates how perceptual factors influence the three stages and lead to regulation flexibility and traffic management measures. This paper focuses on observation and evaluation. With regard to observation, earlier research showed that the awareness among travelers of changes in the transport system is limited, sometimes even leading to perceptions opposite to actual experiences $[5,11]$. Moreover, studies in cognitive sciences show that people are surprisingly bad at detecting even large changes, sometimes leading to change blindness in a large majority of all cases [7]. In a traffic management context, a change could involve an improvement or decline of an existing alternative or the 
introduction of a new alternative, and concern for example the waiting time at traffic lights, average speed or travel time. When it comes to evaluation, the question is whether travelers are able to value a change properly once it has been detected? Previous research showed that travelers have little feeling of absolute value as they focus on relative advantages in relation to a reference point based on previous experiences, compare them locally to the available alternative, and overweigh losses and short term impacts compared to gains and long term impacts $[2,12,13]$. The reference point may be vague and fuzzy, not based on actual experiences, and differ from one traveler to another and from situation to situation [12]. Additionally and related to choice, changes may be outside the area of interest of a traveler as results from satisfying behavior, which states that people are happy with a good solution instead to find the best solution $[3,5]$. This means that travelers tend to minimize their cognitive efforts, and follow simple heuristics to reach decisions which are both satisfactory and sufficient, especially under uncertainty and time constraints $[11,14]$. In addition, decisions and actions of travelers not always correspond with their (perceived) observations. In one study [11], only $12 \%$ of the drivers was able to correctly perceive their experienced travel times, and reversely, $12 \%$ perceived the opposite of their experience.

The objectives of this study were: (1) determine travelers' ability to detect changing traffic intensity and traffic light settings on their trip, and (2) determine how travelers estimate the value of such changes. An internet survey was conducted of which the methodology and results are discussed in this paper. When setting up a survey in the context of decision-making as outlined above, it is important to note that due to the analytical similarity of the perspective of limited awareness and disinterest or indifference toward alternatives, it is hardly impossible to empirically distinguish between the two based on choice outcomes alone [5]. For example, it is impossible to tell from the observation that a traveler responds to a change or not, whether this results from the (in)ability to observe the change or from indifference and disinterest to act upon it. As a result, no elements of route choice were included in the survey, while the survey questions were in particular targeted to collect knowledge about participants' awareness and indifference levels.

\section{METHODOLOGY}

\section{A. Video survey}

In order to systematically assess the effects of small changes in trip attributes on the ability to detect these changes, a video experiment was set-up. The video experiment allowed full control over the traffic conditions and driving behavior. Compared to other methods such as a driving simulator experiment or real-world experiment, the costs are relatively low and the number of participants can be relatively high in a short time period. Even though simulated videos may not be $100 \%$ realistic, this approach avoided any a priori expectations from the participants. Besides it also has a major advantage over classical stated preference surveys, because it would be almost impossible to describe the perception of a change to traffic intensity and traffic light settings without any actual experience. The videos were recorded using the traffic simulation model VISSIM. For all scenarios, the same network and environment were used. It concerned a straight two-lane road with a length of 1 kilometer and three controlled intersections. The maximum speed limit was set to $50 \mathrm{~km} / \mathrm{h}$ and the distance between intersections was 300 meters. Regardless of the scenario, all videos had an equal play time of 1 minute, which means that less distance was covered in the scenarios with a higher travel time. There were two reasons for this decision: (1) to prevent participants using the video play time as an indicator for the traffic performance, and (2) participants lose interest and get distracted if the play time exceeds 1 minute as shown by a trial survey. Finally, the absence of a dashboard and bonnet was compensated by placing a lead vehicle in front of the vehicle of the participant to provide a reasonable reference for position and speed.

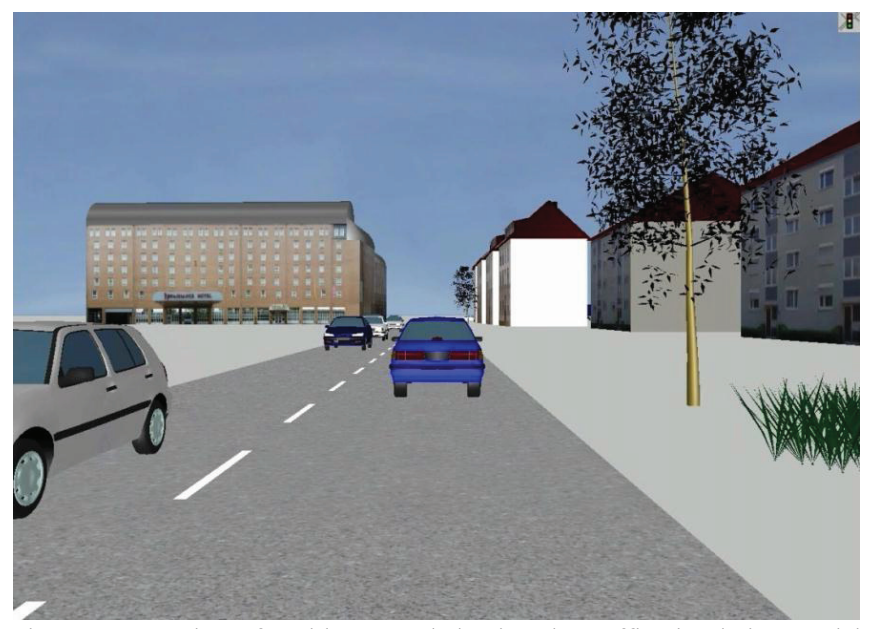

Fig. 1. Screenshot of a video recorded using the traffic simulation model VISSIM. In each scenario, the participants follow the lead vehicle in blue and pass three controlled intersections. Play time of all videos is 1 minute.

\section{B. Driving conditions}

In total 6 different scenarios were used in which traffic intensity and traffic signal settings were varied. Traffic intensities were varied between 300, 600, 900 and 1200 vehicles/hour, while the traffic signal settings could be either synchronized (i.e. green wave) or non-synchronized. Synchronization means that participants experienced a green wave at the intersections 2 and 3, which was only applied in the busy scenarios with traffic intensities of 900 and 1200 vehicles/hour. The 6 scenarios were carefully balanced and ordered across four different surveys in a way that as many scenario combinations were covered, while the participants were randomly assigned to the four surveys. The first video showed the same scenario in all surveys and served as a common reference for further analysis. On average a survey took 15 minutes in which a participant watched 6 videos. Earlier research indicated that these amounts are acceptable $[3,15]$. Consequently, it was not feasible to include all 
possible scenario combinations (i.e. 36). Therefore, a selection has been made intuitively, based on the similarity of scenarios. Figure 2 shows the distribution and order of scenarios across surveys and videos.

\begin{tabular}{|c|c|c|c|c|c|}
\hline & & Survey 01 & Survey 02 & Survey 03 & Survey 04 \\
\hline - Video 01 & & 0 & 0 & 0 & 0 \\
\hline Video 02 & & $\downarrow$ & 4 & 0 & 44 \\
\hline Video 03 & & 44 & 4 & $\downarrow$ & 4 \\
\hline Video 04 & & 4 & $\uparrow$ & 44 & 44 \\
\hline Video 05 & & 0 & $\downarrow$ & 4 & $\downarrow$ \\
\hline Video 06 & & 44 & 44 & 0 & 44 \\
\hline Legend: & $\begin{array}{l}0 \\
4\end{array}$ & $\begin{array}{l}300 \text { veh/h } \\
\text { Non-synchror } \\
600 \text { veh/h } \\
\text { Non-synchror } \\
900 \text { veh/h } \\
\text { Non-synchror }\end{array}$ & $\begin{array}{l}\text { ized signals } \\
\text { ized signals } \\
\text { ized signals }\end{array}$ & $\begin{array}{c}1200 \text { veh } \\
\text { Non-sync } \\
\text { 44 } 900 \text { veh/l } \\
\text { Synchron } \\
441200 \text { veh } \\
\text { Synchron }\end{array}$ & $\begin{array}{l}900 \text { veh/h } \\
\text { Synchronized signals } \\
1200 \text { veh/h } \\
\text { Synchronized signals }\end{array}$ \\
\hline
\end{tabular}

Fig. 2. Overview of distribution and arrangement of scenarios. Four different surveys were prepared with 6 videos each. Video 1 was the same for all surveys. Participants had to compare scenarios as indicated by the arrows on the left side of the Figure.

\section{Experiment procedure}

Participants were invited to take part in the survey by providing them with a link to the webpage of the survey. They were informed that the aim of the survey was to determine travelers' perception of traffic situations, and that they would see 6 videos for which they needed to answer a number of questions. The participants were instructed to imagine as if they drive the route from home to work on a daily basis. First, the participants were asked to provide some personal characteristics. Next, for the first video only a grade for the traffic condition was requested, while for the other videos the participants were presented with the questions discussed earlier. It took about 15 minutes to complete the survey.

\section{Questionnaire}

Besides videos, the survey included a number of questions related to each of videos. The aim of the questions was to determine participants' perception while comparing one scenario to another. In all surveys, the videos 1, 3 and 5 served as a reference for comparison with other videos. The arrows in Figure 2 show which videos were compared with each other. By means of three questions, participants were asked to indicate whether the traffic situation in video 2 had improved as compared to video 1 or not. The first question asked to grade the traffic situation on a scale from 1 to 10 . Secondly, the participants were asked if they thought the situation had improved, worsened or was the same compared to the reference scenario. In case they answered that the scenario had improved or worsened, they were asked to indicate the magnitude of the change on a 5-point scale ranging from 'hardly noticeable' to 'clear difference'. After each question the participant had the possibility to provide additional comments if they desired to. About 20 percent of the participants left one or more remarks, mostly commenting that the purpose of the research was unclear.
This can easily be explained, because a retrospective approach was chosen, which means that participants do not know in advance what will be requested from them [16]. In perception studies it is important that participant act as closely to their normal behavior as possible, without pointing them to certain aspects.

\section{E. Study limitations}

Most importantly and despite all efforts, simulation based videos are very different from real-world driving. Besides, a one minute video cannot capture all elements of an average trip in reality. Besides, participants did not have a clear view on the traffic on their lane like the queues in front. Another disadvantage is the presence of a time indicator in most video players, which allows participants to keep track of time. Perhaps the fact that videos were cut to a 60 seconds length, which means that start and end positions varied making comparison harder, provided some compensation.

\section{RESULTS}

\section{A. Participants}

The research involved a total of 70 completed surveys which were collected in January 2011. Participants were invited by e-mail and internet forums. Although internet forums may guarantee a diverse response, two-third of all responses from these forums appeared to be not useable. In most cases the survey was not completed or the answers were not realistic. The majority of the participants was male (92\%), of age between 18-30 years old (61\%), and in possessing of a valid driving license (90\%). Finally, there was an equal distribution over profession types and frequent and less-frequent drivers were well mixed. Statistical analysis did not reveal significant differences between different participant categories.

\section{B. Loss aversion}

To study if participants' value of outcome is different in case of losses compared to gains, the stated magnitude of the improvement or decline has been mapped against the corresponding grade change as shown in Figure 3 . The value function shows a nearly perfect linear relation, which suggest that on average participants were rational and hardly discriminated between positive and negative experiences. Polynomial functions did not provide a better fit with the data. This was unexpected and not in line with prospect theory as discussed before, which suggested large and irrational differences between gains and losses.

\section{Reference point}

To study the effect of previous experiences and the reference point, four cases were taken from the data set which involves a base scenario followed by a different scenario and next returning to the base scenario again (see Table I). It was assumed that based on the grades of the participants for the subsequent tasks, reference point and 
anchor effects could be studied. Effects were expected to be biggest when the difference between the scenarios was largest. Results are shown in Table II.

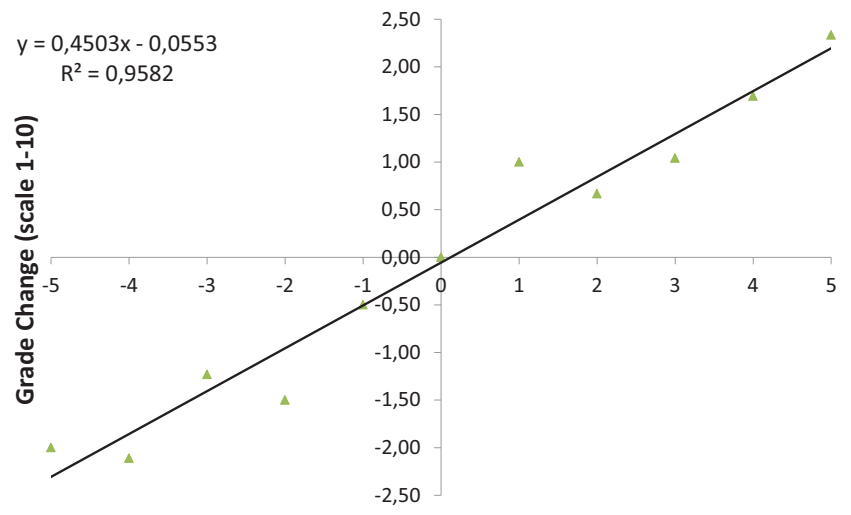

Stated Improvement/Decline (scale 1-5)

Fig. 3. Absolute grade changes from the previous scenario to the current on a scale of 1 to 10 (y-axis), as opposed to participants' stated improvement (i.e. positive or gain) or decline (i.e. negative or loss) of the traffic conditions on an interval scale ( $1=$ hardly noticeable, $5=$ clear difference), as compared to the previous scenario (x-axis). A linear value function with grade changes being proportional to the state change indicates perfect rational perception.

Using an one-sided t-test for unequal variances the difference between scenarios was tested for significance (i.e. $\mathrm{P}<0,05)$, for which the results are shown in Table II. They show that for none of four cases, scenarios 1 and 3 are significantly different. In other words, the participants valued the first and last scenario statistically equal. Figure 4 graphically illustrates the small differences. For the cases which involved a change in only the traffic intensity (i.e. cases 1 and 3), grades were not significantly different.

TABLE I

SCENARIO SEQUENCE FOR REFERENCE POINT ANALYSIS

\begin{tabular}{|c|c|c|c|}
\hline & Scenario 1 & Scenario 2 & Scenario 3 \\
\hline Case 1 & $\begin{array}{c}300 \mathrm{veh} / \mathrm{h} \\
\text { non-synchronized }\end{array}$ & $\begin{array}{c}1200 \mathrm{veh} / \mathrm{h} \\
\text { non-synchronized }\end{array}$ & $\begin{array}{c}300 \mathrm{veh} / \mathrm{h} \\
\text { non-synchronized }\end{array}$ \\
\hline Case 2 & $\begin{array}{c}300 \mathrm{veh} / \mathrm{h} \\
\text { non-synchronized }\end{array}$ & $\begin{array}{c}1200 \mathrm{veh} / \mathrm{h} \\
\text { synchronized }\end{array}$ & $\begin{array}{c}300 \mathrm{veh} / \mathrm{h} \\
\text { non-synchronized }\end{array}$ \\
\hline Case 3 & $\begin{array}{c}600 \mathrm{veh} / \mathrm{h} \\
\text { non-synchronized }\end{array}$ & $\begin{array}{c}1200 \mathrm{veh} / \mathrm{h} \\
\text { non-synchronized }\end{array}$ & $\begin{array}{c}600 \mathrm{veh} / \mathrm{h} \\
\text { non-synchronized }\end{array}$ \\
\hline Case 4 & $\begin{array}{c}600 \mathrm{veh} / \mathrm{h} \\
\text { non-synchronized }\end{array}$ & $\begin{array}{c}1200 \mathrm{veh} / \mathrm{h} \\
\text { synchronized }\end{array}$ & $\begin{array}{c}600 \mathrm{veh} / \mathrm{h} \\
\text { non-synchronized }\end{array}$ \\
\hline
\end{tabular}

TABLE II

GRADES AND T-TESTS FOR SCENARIO SEQUENCES

\begin{tabular}{ccccc}
\hline \hline & Case 1 & Case 2 & Case 3 & Case 4 \\
\hline Grade scenario 1 & 6,15 & 6,15 & 6,33 & 6,33 \\
Grade scenario 2 & 5,86 & 6,91 & 5,78 & 7,32 \\
Grade scenario 3 & 6,00 & 6,33 & 6,15 & 6,12 \\
t-test 1 versus 2 & $\mathrm{P}=0,21$ & $\mathrm{P}=0,01$ & $\mathrm{P}=0,13$ & $\mathrm{P}<0,01$ \\
t-test 2 versus 3 & $\mathrm{P}=0,37$ & $\mathrm{P}=0,13$ & $\mathrm{P}=0,14$ & $\mathrm{P}<0,01$ \\
t-test 1 versus 3 & $\mathrm{P}=0,35$ & $\mathrm{P}=0,34$ & $\mathrm{P}=0,24$ & $\mathrm{P}=0,22$ \\
\hline \hline
\end{tabular}

However, for the same cases but including the introduction of traffic light synchronization (i.e. cases 2 and 3 ), statistical difference were obtained. In case 4, with slightly higher traffic intensity in scenario 1 and 3 , the effect is stronger compared to case 2. This suggests that the effect of traffic light synchronization is more noticeable to travelers when their reference traffic intensity is higher. Overall, these results indicate that it was easier for participants to notice and value differences in traffic light control than in traffic intensities.

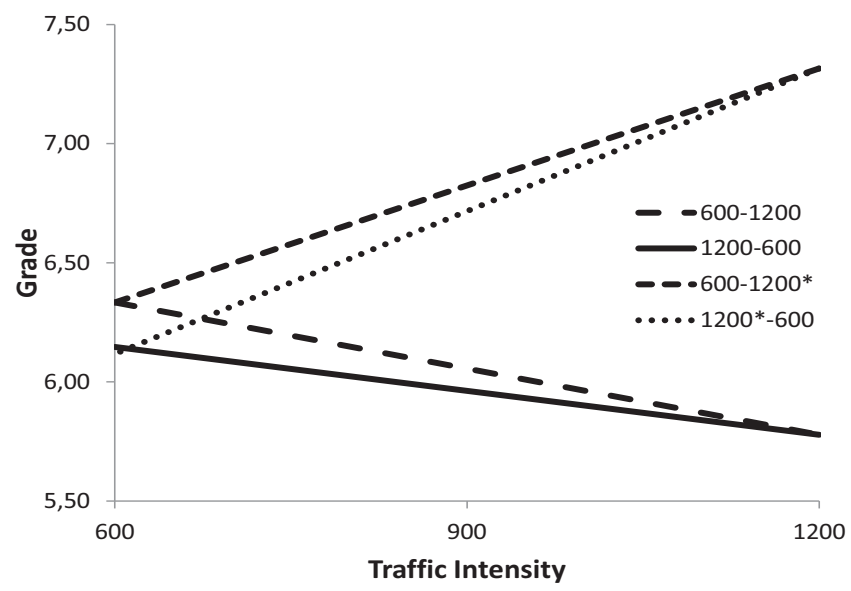

Fig. 4. Grades for scenario sequence 600 to 1200 and back, 1200 to 600 vehicles per hour, for 1200 vehicles per hour with (*) and without traffic light synchronization.

\section{Perception vs. experiences}

To analyze how perception is influenced by objective experiences, four scenario types were shown to the participants: (1) those involving a decrease in traffic intensity, (2) those involving an increase in traffic intensity, (3) those involving an increase in traffic intensity plus the introduction of traffic light synchronization, and (4) those involving a decrease in traffic intensity plus the removal of traffic light synchronization. The first type is regarded as an improvement and the second type as a decline, which means that for these types it can be determined whether the perception of participants was 'correct' or not. For the last two types it is indecisive if it involves an improvement or decline, these were particularly included to analyze the effect of changes in traffic light control as compared to traffic intensities. Results are shown in Table III and Table IV.

TABLE III

PERCEPTION VERSUS EXPERIENCES TRAFFIC INTENSITY CHANGES

\begin{tabular}{lcccc}
\hline \hline & Intensity $\downarrow$ & Intensity $\uparrow$ & & Sum \\
\hline Improve & $33(29 \%)$ & $\mathbf{1 9}(\mathbf{2 3} \%)$ & Correct & $72(37 \%)$ \\
Decline & $36(32 \%)$ & $\mathbf{3 9}(\mathbf{4 6} \%)$ & Opposite & $55(28 \%)$ \\
Identical & $44(39 \%)$ & $26(31 \%)$ & Indifferent & $70(36 \%)$ \\
Total & 113 & 84 & & 197 \\
\hline \hline
\end{tabular}

* Bold cells are significant effects; marked cells are perceptions that correspond with the actual change and therefore 'correct'. The last column shows the sum of correct, opposite and indifferent perceptions. 
TABLE IV

PERCEPTION VERSUS EXPERIENCES TRAFFIC INTENSITY CHANGES AND TRAFFIC LIGHT SYNCHRONIZATION INTRODUCTION

\begin{tabular}{|c|c|c|c|c|}
\hline & $\begin{array}{c}\text { Intensity } \downarrow \\
\text { Synch. }+\end{array}$ & $\begin{array}{c}\text { Intensity } \uparrow \\
\text { Synch. - }\end{array}$ & & Sum \\
\hline Improve & $68(63 \%)$ & $3(8 \%)$ & Correct & $93(65 \%)$ \\
\hline Decline & $25(23 \%)$ & $25(69 \%)$ & Opposite & $28(19 \%)$ \\
\hline Identical & $15(14 \%)$ & $8(23 \%)$ & Indifferent & $23(16 \%)$ \\
\hline Total & 108 & 36 & & 144 \\
\hline
\end{tabular}

Participants were asked to indicate if they thought the traffic situation had improved, worsened or was identical compared to the reference scenario presented in one of the two previous videos. The data have been tested on significance using a binomial test. Scenario types 1 and 2 only involve traffic intensity changes, whereas scenario types 3 and 4 also include traffic light synchronization. When comparing scenario type 1 with scenario type 2 , the data suggests that participants were better able to detect increases in traffic intensities (i.e. type 2 and involving loss), than a decrease in traffic intensities (i.e. type 1 and involving gain): $46 \%$ versus $29 \%$ respectively. More specifically, no significant effects were found for scenario type 1, while for type 2 significant effects were found for correct and opposite observations. Note that the absence of an effect means that for those types of change, the change is unlikely to be noticed. Most notable are the percentages of participants with the 'wrong' perception (i.e. the non-grey cells). On average, no significant effects were found for changes related to traffic intensity alone. The results did not differ for larger absolute differences between scenarios, for example a difference of $300 \mathrm{veh} / \mathrm{h}$ compared to a difference of 900 $\mathrm{veh} / \mathrm{h}$.

Results of scenario types 3 and 4 show that the involvement of traffic light synchronization makes a big difference. More effects were found for these scenarios, see Table 4. Significant effects were found for almost all observations. For both scenario types, a clear majority of participants regarded the presence of traffic light synchronization as an improvement: $63 \%$ and $69 \%$ respectively. Besides, the parallel changes in traffic intensity could not compensate for the fact that traffic light synchronization was introduced or removed. For example, $69 \%$ of all participants regarded the removal of the green wave as a decline, despite a decrease in traffic intensity. Moreover, constant changes in traffic intensity but combined with traffic light synchronization, had a much are larger effect compared to changes in traffic intensities alone. For example, $63 \%$ of the participants perceived the introduction of the green wave as an improvement even though the traffic intensity increased. In these scenario types, on average only $35 \%$ of the participants missed the direction of change, which is much lower compared to scenario types 1 and 2 .
Nonetheless, no effect was found for opposite observations.

\section{E. Confidence}

Finally the participants' confidence level with regard to what they perceived was studied. For this analysis the 5point scale to indicate the magnitude of differences was used. It was assumed that people would state a bigger difference if they were more confident, while a smaller standard deviation across answers given by the population is assumed to indicate a higher general confidence level for that particular scenario. Table 5 summarizes the results, in which 1 stands for 'clear difference' and 5 represents 'hardly noticeable'. In general it was found that participants tended to avoid the outer answers of the scale, which means all answers were very similar despite the differences between scenarios. Therefore, no significant effects were found.

TABLE V

STATED MAGNITUDE OF CHANGES

\begin{tabular}{lcccc}
\hline \hline & Type 1 & Type 2 & Type 3 & Type 4 \\
\hline Improve ave. & 3.08 & 3.41 & 2.53 & 3.00 \\
Improve stdev. & 0.83 & 0.87 & 0.78 & $\mathrm{n} / \mathrm{a}$ \\
Decline ave. & 3.02 & 2.97 & 2.55 & 2.61 \\
Decline stdev. & 1.03 & 0.93 & 0.96 & 0.61 \\
\hline \hline
\end{tabular}

Regarding the figures as indicative only, participants seem to be more confident about observing changes which negatively affect their situation as compared to scenarios which improve their situation. Furthermore, both the averages and standard deviations confirm that participants were more confident about scenarios involving traffic light synchronization.

\section{DISCUSSION AND CONCLUSIONS}

Based on the survey results it can be concluded that travelers are not able to observe all changes in traffic conditions. Moreover, road users are more sensitive to changes in traffic light settings than to changes in traffic intensities. Apparently, traffic light settings have the attention of travelers, in any case much more than changes in traffic intensities. Despite the relative easy setup of the survey, a number of interesting results have been obtained which deserve to be explored in more depth. For example, the results provide insufficient evidence to speak of systematic and predictable effects related to behavioral imperfections and irrationality. Mostly this is due to the selected setup of the survey and the number of participants. The relative complex methodology which combines multiple scenarios, multiple surveys and a variety of answering options requires a much larger response than was obtained in this study. Consequently, the survey results provide an indication of the direction of the effects, but only a part of the effects is significant. This was most apparent in the reference point analysis for changes in traffic intensities. 
Nevertheless, again it is important to note that in the context of change detection, the absence of significant effects, in fact offers very valuable knowledge. In such a case, it means that the event of change detection is random and uniformly distributed across 'correct detection', 'opposite detection' and 'no detection'. In other words, the related change goes unnoticed by roughly one-third of the travelers, and noticed but incorrectly valued by another one-third.

With regard to the results on loss aversion it is interesting to note that during the process of data sorting it appeared that the value function is nearly identical to the one reported in literature on prospect theory [4], i.e. concave in gains and convex in losses, if the outliers were not removed from the data set. This suggests that the data which are normally removed from the data set could represent the irrational part of the participants' perception. However, the available dataset was too small to determine whether these outliers were systematic and to accept this hypothesis. In general, most of the results obtained in this study are in line with the theories derived from prospect theory and other studies discussed earlier. For example the results on perception versus experiences comparisons confirm the existence of opposite perceptions, while in general the majority of travelers do not notice changes (correctly). Besides, confidence levels underline that travelers are likely to detect decrease in performance (i.e. losses) sooner than improvements in performance (i.e. gains).

To conclude, the findings from this survey show that there is sufficient ground for the concept of regulation flexibility. More research is needed on this topic to understand the effect of other changes in traffic and traffic conditions, and to take into account the relative difference between changes of different attributes. For example, it is arguable that in absolute terms, changes to traffic light synchronization are much larger than small changes to traffic intensity. In other words, the underlying question is whether same-size changes to different attributes result in equal sensitivity and change detection effects. From this study it can be concluded that the setting of traffic lights, in particular synchronization, is a dominant variable, while the variability of the traffic intensity has a relative low weight and can be higher than the steps of 300 vehicles used in this study. Of additional interest are for example speed and route choice variables, while the influence of learning effects and information provisioning could be studied in parallel. For future research it is recommended to choose simpler experimental design with fewer variables to increase the usefulness of the data. Furthermore, given the drawbacks and limitations of data collection in simulated environments it is recommended to use real-world videos or set up field experiments and study the correlation between subjective data (e.g. survey) and objective data (e.g. actual measurements). Finally, knowledge on change detection and regulation flexibility needs to be translated to policy and strategy definitions which explicitly state which variables (not) to vary in relation to certain base conditions and traffic management measures.

\section{ACKNOWLEDGMENT}

This research is part of a PhD. research funded by Peek Traffic and the University of Twente. The survey was conducted as an assignment for a master course on Intelligent Transportation Systems at the University of Twente. The authors would like to thank all their respondents for their time and valuable answers.

\section{REFERENCES}

[1] E. Avineri, "'Choice Architecture' and the Design of Advanced Traveler Information Systems (ATIS)," presented at the Proceedings of the 13th IEEE - ITS Conference - Workshop on Traffic Behavior, Modeling and Optimization, Madeira 2010.

[2] D. Ariely, Predictably Irrational - Revised and expanded edition. London: HarperCollingsPublishers, 2009.

[3] E. A. I. Bogers, F. Viti, and S. P. Hoogendoorn, "Joint modeling of advanced travel information service, habit, and learning impacts on route choice in laboratory simulator experiments," Transportation Research Record, vol. No. 1926, pp. 189-197, 2005.

[4] E. Avineri and J. N. Prashker, "Violations of expected utility theory in route-choice stated preferences," Transportation Research Record, vol. No. 1894, pp. 222-229, 2004.

[5] C. G. Chorus and H. J. P. Timmermans, "Measuring user benefits of changes in the transport system when traveler awareness is limited," Transportation Research Part A: Policy and Practice, vol. 43, pp. 536-547, 2009.

[6] J. D. Vreeswijk, E. C. V. Berkum, and B. v. Arem, "Flexible traffic management based on bounded rationality and indifference bands," presented at the 2nd Symposium on Model and Technologies for Intelligent Transportation Systems, Leuven, Belgium, 2011.

[7] M. Martens, "Change detection in traffic: where do we look and what do we perceive?," Transportation Research Part F: Traffic Psychology and Behaviour, vol. 14F, pp. 240-250, 2011.

[8] P. M. Pearson and E. G. Schaefer, "Toupee or not toupee? The role of instructional set, centrality, and relevance in change blindness," Visual Cognition, vol. Vol. 12, pp. pp. 1528-1543, 2005.

[9] J. Bie and H. K. Lo, "Stability and attraction domains of traffic equilibria in a day-to-day dynamical system formulation," Transportation Research Part B: Methodological, vol. 44, pp. 90107, 2010.

[10] J. D. Vreeswijk, E. C. Van Berkum, H. A. Van der Vliet, and B. Van Arem, "Effective strategies for energy efficient network management," presented at the Proceedings of the 13th International IEEE - ITS Conference, Madeira 2010.

[11] A. Tawfik, H. Rakha, and S. Miller, "Examining drivers experiences, perceptions, and choices in route choice behavior," presented at the Proceedings of the 13th IEEE - ITS Conference - Workshop on Traffic Behavior, Modeling and Optimization, Madeira, 2010.

[12] E. Avineri and P. H. L. Bovy, "Identification of parameters for a prospect theory model for travel choice analysis," Transportation Research Record, vol. No. 2082, pp. 141-147, 2008.

[13] A. Tversky and D. Kahnemann, "Advances in prospect theory: Cumulative representation of uncertainty," Journal of Risk and Uncertainty, vol. vol. 9, pp. pp. 195-230, 1992.

[14] K. K. Srinivasan and H. S. Mahmassani, "Role of congestion and information in trip-makers' dynamic decision processes experimental investigation," Transportation Research Record, pp. 44-52, 1999.

[15] B. Van der Bijl, J. D. Vreeswijk, J. Bie, and E. C. Van Berkum, "The perception and acceptance of waiting at signalized intersection by car drivers," presented at the In preparation for the 14th International IEEE - ITS Conference, Washington D.C., 2011.

[16] K. K. Pecheux, "User perception of time-based level-of-service criteria for signalized intersections," Department of Civil Engineering, Pennsylvania State University, 2000. 